\title{
Glatiramer acetate does not protect from acute ischemic stroke in mice
}

Peter Kraft ${ }^{1,2+}$, Kerstin Göbel ${ }^{3 \dagger}$, Sven G Meuth ${ }^{3,4}$ and Christoph Kleinschnitz ${ }^{1 *}$

\begin{abstract}
Background: The role of the immune system in the pathophysiology of acute ischemic stroke is increasingly recognized. However, targeted treatment strategies to modulate immunological pathways in stroke are still lacking. Glatiramer acetate is a multifaceted immunomodulator approved for the treatment of relapsing-remitting multiple sclerosis. Experimental studies suggest that glatiramer acetate might also work in other neuroinflammatory or neurodegenerative diseases apart from multiple sclerosis.

Findings: We evaluated the efficacy of glatiramer acetate in a mouse model of brain ischemia/reperfusion injury. 60 min of transient middle cerebral artery occlusion was induced in male C57BI/6 mice. Pretreatment with glatiramer acetate (3.5 mg/kg bodyweight) $30 \mathrm{~min}$ before the induction of stroke did not reduce lesion volumes or improve functional outcome on day 1 .

Conclusions: Glatiramer acetate failed to protect from acute ischemic stroke in our hands. Further studies are needed to assess the true therapeutic potential of glatiramer acetate and related immunomodulators in brain ischemia.
\end{abstract}

Keywords: Glatiramer acetate, Stroke, Inflammation, Neurodegeneration

\section{Findings}

For many years ischemic stroke has been regarded as a mere thrombo-embolic disease. However, there is increasing evidence that the immune system is also critically involved in stroke occurrence and development [1,2]. Brain ischemia triggers rapid activation of cerebral endothelial cells and the release of danger signals from dying neuronal tissues. As a consequence, different cell adhesion receptors are upregulated and soluble chemoattractants such as chemokines and cytokines are secreted that guide the targeted invasion of innate immune cells (neutrophils, macrophages) to the sites of tissue damage [2]. These peripheral immune cells in concert with resident cell populations like microglia or astrocytes produce a potpourri of potentially harmful mediators (e.g. reactive oxygen species, degrading enzymes) and this sterile inflammation further perpetuates the ischemic cascade. Interestingly, $\mathrm{T}$ lymphocytes, which belong to the adaptive immune

\footnotetext{
* Correspondence: christoph.kleinschnitz@mail.uni-wuerzburg.de

${ }^{\dagger}$ Equal contributors

'Department of Neurology, University Hospital Würzburg, Josef-Schneider-Str. 11, 97080 Würzburg, Germany

Full list of author information is available at the end of the article
}

system, can likewise foster ischemic brain damage. $\operatorname{Ragl}^{-/-}$ mice lacking functional $\mathrm{T}$ lymphocytes develop dramatically smaller brain infarctions and less severe functional deficits after transient middle cerebral artery occlusion [3-5]. Of note, distinct $\mathrm{T}$ cell subsets for instance regulatory T cells (Treg) or $\gamma \delta$ T cells exist that are particularly harmful in stroke [5]. Given that the immune system is of significant relevance for the pathophysiology of acute ischemic stroke, it seems reasonable to evaluate the safety and efficacy of specific immunomodulatory compounds in terms of stroke prevention and treatment.

Glatiramer acetate (GA) is a synthetic peptide that consists of four amino acids in a fixed molar residue ratio [6]. The substance for many years is in clinical use for the treatment of relapsing-remitting multiple sclerosis and has demonstrated to suppress experimental allergic encephalomyelitis (EAE), the most common animal model of multiple sclerosis [6]. The exact mode of action of GA in autoimmune neuroinflammation is still unclear but among other things is based on its ability to inhibit Th1 proinflammatory cytokines and to induce Th2 cell and Treg activation or to reduce monocyte reactivity [7]. Direct neuroprotective effects mediated for instance by 
brain-derived neurotrophic factor (BDNF) may also play a role [8]. However, the therapeutic potential of GA in ischemic stroke is unclear and previous studies in rodents produced controversial results $[9,10]$. Here, we investigated the effect of GA on stroke outcome in a wellestablished mouse model of brain ischemia/reperfusion injury.

All experiments were approved by the respective institutional (University of Würzburg, Germany; University of Münster, Germany) and governmental authorities (Regierung von Unterfranken and Landesamt für Natur, Umwelt und Verbraucherschutz Nordrhein-Westfalen). Six to eight week old male $\mathrm{C} 57 \mathrm{Bl} / 6$ mice weighing 20-25 g were used in the study which was conducted in accordance with the recently published ARRIVE guidelines (http://www.nc3rs.org/ARRIVE). 60 min of transient middle cerebral artery occlusion (tMCAO) using a monofilament was performed as described [11]. This model mounts a strong and immediate local inflammatory response in the ischemic brain [12]. GA (TEVA Pharma, $3.5 \mathrm{mg} / \mathrm{kg}$ bodyweight) [13] or vehicle ( $\mathrm{NaCl} 0.9 \%)$ were injected intravenously $30 \mathrm{~min}$ before tMCAO. Animals were randomly assigned to the operators by an independent person not involved in data acquisition and analysis.
We performed surgery and evaluation of all read-out parameters while being blinded to the experimental groups. Infarct volumes were calculated from coronal brain slices stained with 2,3,5-triphenyltetrazolium chloride (TTC) $24 \mathrm{~h}$ after tMCAO [11]. The Bederson score [14] and the grip test score [15] were assessed to monitor global neurologic function, motor function, and coordination.

Stroke volumes were expressed as mean \pm SEM. Functional outcome parameters were depicted as scatter plots including median with the $25 \%$ percentile and the $75 \%$ percentile given in brackets in the text. For statistical analysis, the GraphPad Prism 5.0 software package (GraphPad Software) was used. Data were tested for Gaussian distribution with the D'Agostino and Pearson omnibus normality test and then analyzed by unpaired, two-tailed Student's t-test or Mann Whitney U test. P values $<0.05$ were considered statistically significant.

Prophylactic administration of GA did not alter stroke outcome (Figure 1). Stroke volumes on day 1 after $60 \mathrm{~min}$ tMCAO were similar between GA-treated mice and vehicletreated controls $\left(66.4 \mathrm{~mm}^{3} \pm 37.3 \mathrm{~mm}^{3}\right.$ vs. $76.7 \mathrm{~mm}^{3} \pm$ $31.0 \mathrm{~mm}^{3}, \mathrm{n}=7, \mathrm{p}>0.05$ ) (Figure 1A). Accordingly, no differences in functional outcome parameters were observed (Bederson score: $3.0[2.0,3.0]$ vs $3.0[1.0,3.0]$,
A

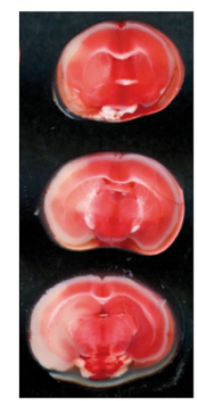

GA
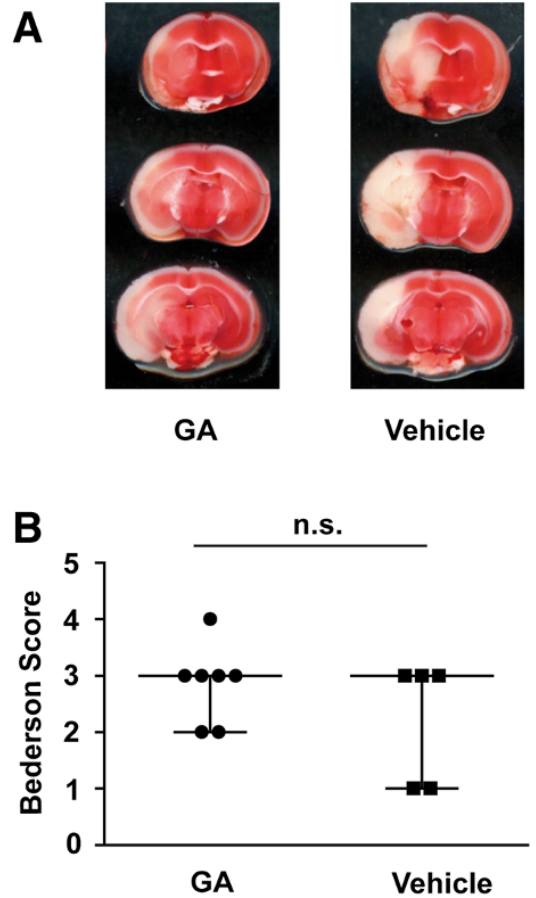

Vehicle

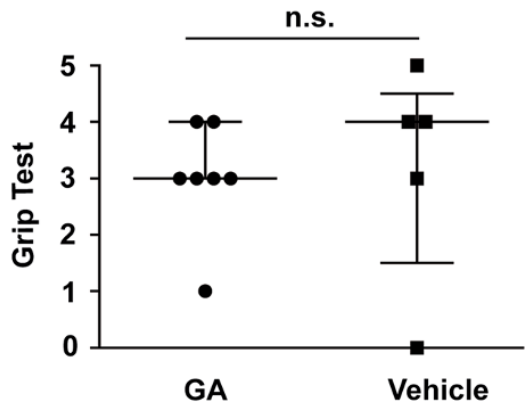

Figure 1 Glatiramer acetate (GA) does not protect from acute brain ischemia/reperfusion injury. (A) (Left panel) Representative 2,3, 5-triphenyltetrazolium chloride stains of 3 corresponding coronal brain sections of a GA-treated mouse and a vehicle-treated mouse sacrificed on day 1 after transient middle cerebral artery occlusion (tMCAO). GA was applied $30 \mathrm{~min}$ before tMCAO at a dosage of $3.5 \mathrm{mg} / \mathrm{kg}$ bodyweight. Stroke sizes appeared to be similar between the two groups and this was confirmed by infarct volumetry (right panel) ( $n=7 /$ group). (B) GA did not improve functional outcome on day 1 after tMCAO. The Bederosn score (left panel) and the grip test (right panel) did not differ between GA-treated mice and vehicle-treated controls ( $n=5-7 /$ group). Unpaired, 2-tailed Student's $t$ test (infarct volumes) or Mann-Whitney Test (Bederson score, grip test); n.s. $=$ not significant. 
$\mathrm{n}=5-7, \mathrm{p}>0.05$; Grip test: $3.0[3.0,4.0]$ vs $4.0[1.5,4.5]$, $\mathrm{n}=5-7, \mathrm{p}>0.05$ ) (Figure 1B).

The present study failed to demonstrate a protective effect of GA in experimental stroke in mice even when administered in a prophylactic setting. This is in line with a previous investigation showing that GA does not reduce stroke volumes or functional deficits on day 3 and day 7 after transient or permanent MCAO despite a downregulation of pro-inflammatory cytokines [10]. In contrast, Ibarra et al. [9] reported that GA exerts beneficial effects on stroke outcome both histologically and clinically after tMCAO in rats when applied 30 min after reperfusion. However, in this study GA-induced neuroprotection became manifest not before day 7 . The exact reasons for these discrepant findings are unclear at present but differences in the stroke models (transient vs permanent) or animal species (rat vs mice) used certainly play a role. In addition, the dosing regimens including the time of GA-application (prophylactic vs therapeutic) differed between the two ancestor studies and ours.

GA has already been tested in other neurodegenerative disease models apart from stroke and EAE but again with divergent results [16]. While GA protected from axonal and neuronal degeneration after optic nerve crush $[17,18]$ or in a mouse model of Alzheimer's disease [19], the substance was ineffective in animal models of amyotrophic lateral sclerosis [20,21]. However, even if GA might mediate some neuroprotection in primarily neurodegenerative diseases its application in ischemic stroke appears less promising.

Several studies already exist that addressed the therapeutic potential of other immunomodulatory agents in models of ischemic stroke. Treatment with FTY720, which sequesters $\mathrm{T}$ lymphocytes within their lymphoid organs [22] and which is also approved for the treatment of multiple sclerosis, protects from ischemic neurodegeneration by reducing the interplay between thrombotic and inflammatory processes ('thrombo-inflammation') in the cerebral microvasculature [11]. However, other modes of FTY720 action such as blood-brain barrier stabilization, reduction of immune cell infiltration or direct neuroprotection might also play a role $[23,24]$. Similarly, blocking of the very late antigen-4/vascular adhesion molecule- 1 axis by injecting a monoclonal antibody against CD49d (the murine equivalent of natalizumab) has been shown to shield the brain against deleterious neuroinflammation after stroke in rodents $[25,26]$ although an identical approach was ineffective in our hands (unpublished observations).

Our study has several limitations. First, we only focused on the outcome at a very early stage after stroke (day 1 ). Therefore, we cannot exclude neuroprotective effects of GA that become operative only at more advanced stages of infarct development [9]. Moreover, different GA dosing or application regimes could have produced different results. Finally, we refrained from conducting profound mechanistic studies since we did not find an obvious phenotype in GA-treated animals.

Taken together, our study failed to confirm a protective effect of GA in acute ischemic stroke in mice. Nevertheless, the concept of immunomodulation in brain ischemia is still tempting given the promising reports on other immunomodulatory agents. Further studies in relevant disease models are warranted.

\section{Competing interests}

The authors declare that they have no competing interests.

\section{Authors' contributions}

PK and KG performed the experiments and drafted the manuscript. SGM and CK conceived and funded the entire study and wrote the manuscript. All authors read and approved the final manuscript.

\section{Acknowledgements}

We thank Melanie Glaser and Daniela Urlaub for excellent technical assistance. This publication was funded by the German Research Foundation (DFG) and the University of Würzburg in the funding programme Open Access Publishing.

\section{Author details}

${ }^{1}$ Department of Neurology, University Hospital Würzburg, Josef-Schneider-Str. 11, 97080 Würzburg, Germany. ${ }^{2}$ Institute for Clinical Epidemiology and Biometry and Comprehensive Heart Failure Centre, University of Würzburg, Würzburg, Germany. ${ }^{3}$ Department of Neurology, University of Münster, Albert-Schweitzer-Campus 1, Gebäude A1, Westturm, Ebene 0548149 Münster, Germany. ${ }^{4}$ Institute of Physiology - Neuropathophysiology, University of Münster, Münster, Germany.

Received: 17 February 2014 Accepted: 18 February 2014 Published: 27 February 2014

\section{References}

1. Magnus T, Wiendl H, Kleinschnitz C: Immune mechanisms of stroke. Curr Opin Neurol 2012, 25:334-340.

2. ladecola C, Anrather J: The immunology of stroke: from mechanisms to translation. Nat Med 2011, 17:796-808.

3. Yilmaz G, Arumugam TV, Stokes KY, Granger DN: Role of T lymphocytes and interferon-gamma in ischemic stroke. Circulation 2006, 113:2105-2112.

4. Kleinschnitz C, Schwab N, Kraft P, Hagedorn I, Dreykluft A, Schwarz T, Austinat M, Nieswandt B, Wiendl H, Stoll G: Early detrimental T-cell effects in experimental cerebral ischemia are neither related to adaptive immunity nor thrombus formation. Blood 2010, 115:3835-3842.

5. Kleinschnitz C, Kraft P, Dreykluft A, Hagedorn I, Göbel K, Schuhmann MK, Langhauser F, Helluy X, Schwarz T, Bittner S, Mayer CT, Brede M, Varallyay C, Pham M, Bendszus M, Jakob P, Magnus T, Meuth SG, Iwakura Y, Zernecke A, Sparwasser T, Nieswandt B, Stoll G, Wiendl H: Regulatory T cells are strong promoters of acute ischemic stroke in mice by inducing dysfunction of the cerebral microvasculature. Blood 2013, 121:679-691.

6. Dhib-Jalbut S: Mechanisms of action of interferons and glatiramer acetate in multiple sclerosis. Neurology 2002, 58:S3-9.

7. Lalive PH, Neuhaus O, Benkhoucha M, Burger D, Hohlfeld R, Zamvil SS, Weber MS: Glatiramer acetate in the treatment of multiple sclerosis: emerging concepts regarding its mechanism of action. CNS Drugs 2011, 25:401-414.

8. Kala M, Miravalle A, Vollmer T: Recent insights into the mechanism of action of glatiramer acetate. J Neuroimmunol 2011, 235:9-17.

9. Ibarra A, Avendaño H, Cruz Y: Copolymer-1 (Cop-1) improves neurological recovery after middle cerebral artery occlusion in rats. Neurosci Lett 2007, 425:110-113. 
10. Poittevin M, Deroide N, Azibani F, Delcayre C, Giannesini C, Levy BI, Pocard M, Kubis N: Glatiramer Acetate administration does not reduce damage after cerebral ischemia in mice. J Neuroimmunol 2013, 254:55-62.

11. Kraft P, Göb E, Schuhmann MK, Göbel K, Deppermann C, Thielmann I, Herrmann AM, Lorenz K, Brede M, Stoll G, Meuth SG, Nieswandt B, Pfeilschifter W, Kleinschnitz C: FTY720 ameliorates acute ischemic stroke in mice by reducing thrombo-inflammation but not by direct neuroprotection. Stroke 2013, 44:3202-3210.

12. Gelderblom M, Leypoldt F, Steinbach K, Behrens D, Choe CU, Siler DA, Arumugam TV, Orthey E, Gerloff C, Tolosa E, Magnus T: Temporal and spatial dynamics of cerebral immune cell accumulation in stroke. Stroke 2009, 40:1849-1857.

13. Scorisa JM, Freria CM, Victorio SC, Barbizan R, Zanon RG, Oliveira AL: Glatiramer acetate treatment increases stability of spinal synapses and down regulates $\mathrm{MHC}$ I during the course of EAE. Int I Biol Sci 2011, 7:1188-202.

14. Bederson JB, Pitts $L H$, Tsuji M, Nishimura MC, Davis RL, Bartkowski H: Rat middle cerebral artery occlusion: evaluation of the model and development of a neurologic examination. Stroke 1986, 17:472-476.

15. Moran PM, Higgins LS, Cordell B, Moser PC: Age-related learning deficits in transgenic mice expressing the 751-amino acid isoform of human beta-amyloid precursor protein. Proc Natl Acad Sci USA 1995, 92:5341-5345.

16. Schwartz M, Bukshpan S, Kunis G: Application of glatiramer acetate to neurodegenerative diseases beyond multiple sclerosis: the need for disease-specific approaches. BioDrugs 2008, 22:293-299.

17. Kipnis J, Yoles E, Porat Z, Cohen A, Mor F, Sela M, Cohen IR, Schwartz M: T cell immunity to copolymer 1 confers neuroprotection on the damaged optic nerve: possible therapy for optic neuropathies. Proc Natl Acad Sci USA 2000, 97:7446-7451.

18. Smirnov I, Walsh JT, Kipnis J: Chronic mild stress eliminates the neuroprotective effect of Copaxone after CNS injury. Brain Behav Immun 2013, 31:177-182.

19. Butovsky O, Koronyo-Hamaoui M, Kunis G, Ophir E, Landa G, Cohen H, Schwartz M: Glatiramer acetate fights against Alzheimer's disease by inducing dendritic-like microglia expressing insulin-like growth factor 1. Proc Natl Acad Sci USA 2006, 103:11784-11789.

20. Habisch HJ, Schwalenstöcker B, Danzeisen R, Neuhaus O, Hartung HP, Ludolph A: Limited effects of glatiramer acetate in the high-copy number hSOD1-G93A mouse model of ALS. Exp Neurol 2007, 206:288-295.

21. Haenggeli C, Julien JP, Mosley RL, Perez N, Dhar A, Gendelman HE, Rothstein JD: Therapeutic immunization with a glatiramer acetate derivative does not alter survival in G93A and G37R SOD1 mouse models of familial ALS. Neurobiol Dis 2007, 26:146-152

22. Chun J, Hartung HP: Mechanism of action of oral fingolimod (FTY720) in multiple sclerosis. Clin Neuropharmacol 2010, 33:91-101.

23. Hasegawa Y, Suzuki H, Sozen T, Rolland W, Zhang JH: Activation of sphingosine 1-phosphate receptor-1 by FTY720 is Neuroprotective after ischemic stroke in rats. Stroke 2010, 41:368-374.

24. Wei Y, Yemisci M, Kim HH, Yung LM, Shin HK, Hwang SK, Guo S, Qin T, Alsharif N, Brinkmann V, Liao JK, Lo EH, Waeber C: Fingolimod provides long-term protection in rodent models of cerebral ischemia. Ann Neurol 2011, 69:119-129.

25. Liesz A, Zhou W, Mracskó É, Karcher S, Bauer H, Schwarting S, Sun L, Bruder D, Stegemann S, Cerwenka A, Sommer C, Dalpke AH, Veltkamp R: Inhibition of lymphocyte trafficking shields the brain against deleterious neuroinflammation after stroke. Brain 2011, 134:704-720.

26. Relton JK, Sloan KE, Frew EM, Whalley ET, Adams SP, Lobb RR: Inhibition of alpha4 integrin protects against transient focal cerebral ischemia in normotensive and hypertensive rats. Stroke 2001, 32:199-205.

doi:10.1186/2040-7378-6-4

Cite this article as: Kraft et al:: Glatiramer acetate does not protect from acute ischemic stroke in mice. Experimental \& Translational Stroke Medicine 2014 6:4.

\section{Submit your next manuscript to BioMed Central and take full advantage of:}

- Convenient online submission

- Thorough peer review

- No space constraints or color figure charges

- Immediate publication on acceptance

- Inclusion in PubMed, CAS, Scopus and Google Scholar

- Research which is freely available for redistribution 